\title{
COMPILER GENERATED SYSTOLIC ARRAYS FOR WAVEFRONT ALGORITHM ACCELERATION ON FPGAS
}

\author{
Betul Buyukkurt \\ Sandbridge Technologies, Inc. \\ Tarrytown, NY 10591 \\ abuyukku@cs.ucr.edu
}

\author{
Walid A. Najjar \\ Computer Science and Engineering \\ University of California Riverside \\ Riverside, CA 92521 \\ najjar@cs.ucr.edu
}

\begin{abstract}
Wavefront algorithms, such as the Smith-Waterman algorithm, are commonly used in bioinformatics for exact local and global sequence alignment. These algorithms are highly computationally intensive and are therefore excellent candidates for FPGA-based code acceleration. However, there is no standard form of these algorithms, they are used in a wide variety of situations with various constraints. It is therefore not practical to have a standard kernel that can be mapped to an FPGA, hence the importance of being able to compile such codes from a high level language. ROCCC is a C to VHDL compiler, which optimizes and parallelizes the most frequently executed kernel loops in applications such as in multimedia, scientific and high-performance computing. In this paper we describe the transformations performed by ROCCC, which transformed the kernel of the Smith-Waterman algorithm into a hardware systolic array that is mapped onto the FPGA on the SGI Altix RASC blade. We report a throughput increase by over 3,000X over a $2.8 \mathrm{GHz}$ Xeon.
\end{abstract}

\section{INTRODUCTION}

Fast sequence alignment computation for DNA sequences has become one of the most challenging computational problems of the decade due to the exponential growth in the size of the biosequence databases. To keep up with this growth, heuristic methods have been adopted in place of exact ones for sequence alignment algorithms to perform faster but potentially inaccurate searches. There are many instances, however, when an exact search must be done at the expense of very large compute times. Furthermore, heuristic search algorithms are known to perform very badly on short strings and it is very common to have sequencing machines generate short strings of 30 to 40 base pairs.

Wavefront algorithms, such as the Smith-Waterman [1] algorithm (shown in Figure 1), are commonly used in bioinformatics for exact local and global sequence alignment. These computations are extremely regular, highly computationally intensive and are applied to a very large set of data. They are excellent candidates for hardware code acceleration. Mapping such computations to a circuit can drastically improve its efficiency as compared to running it on a traditional microprocessor. Wavefront algorithms are usually computed in software

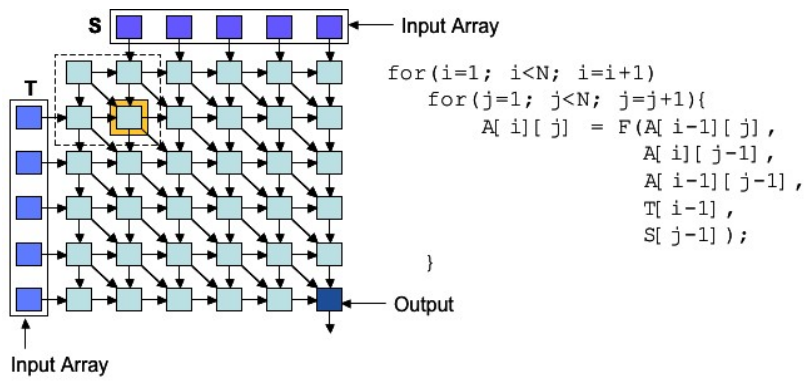

Fig. 1. Wavefront code for sequence alignment

using a 2D matrix where the enclosing loop advances in a fixed direction to compute the current cell using neighboring values computed in previous iterations. A fast implementation of the same algorithm in hardware would be a systolic array on which the computations along the back diagonal are implemented.

In this paper we describe a set of compiler transformations and code generation steps that implement a systolic array on an FPGA, in VHDL, starting from its loop nest expressed in C. These transformations are developed within the context of the ROCCC (Riverside Optimizing Compiler for Configurable Computing) tool set [2]. We evaluate our systolic array generation approach using the Smith-Waterman[1] algorithm which is commonly used in DNA and protein string matching. The generated systolic array was mapped on to the Xilinx Virtex 4 LX200 of the SGI RASC RC100 Blade in the Altix 4700 multiprocessor. The results show a throughput increase larger than $3,000 \mathrm{X}$ over a $2.8 \mathrm{GHz}$ Xeon using a 1,024 element systolic array.

\section{ROCCC OVERVIEW}

ROCCC is a C to RTL VHDL compilation framework for mapping application programs to FPGAs. The focus of ROCCC is generating highly parallel and optimized circuits rather than statement-by-statement translation of C programs to VHDL.

ROCCC is not designed to compile a whole program. In order to identify the kernel codes, ROCCC only requires the user to mark the candidate loop-nest by placing it between 


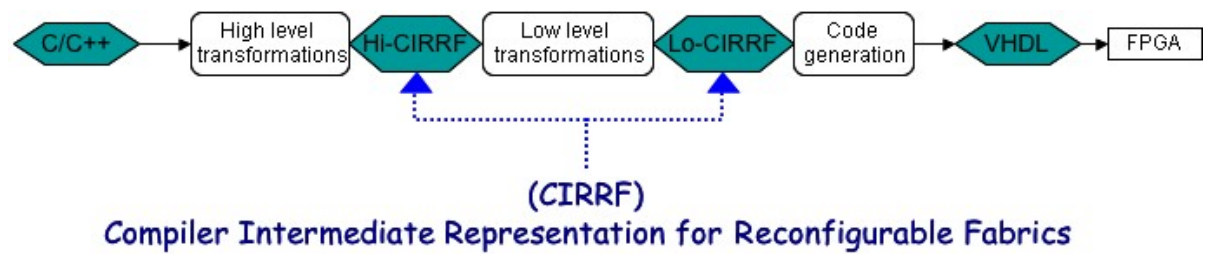

Fig. 2. ROCCC Framework

begin_hw() and end_hw() calls. ROCCC also does not compile arbitrary $\mathrm{C}$ code. The subset of $\mathrm{C}$ that is compilable by ROCCC is defined as follows: no pointers, no break or continue statements, simple for loop headers where the loop counter of each loop level iterates from some lower bound to some upper bound in compile time determinable steps, and that all array index expressions are in form

loop_counter \pm a_compile_time_constant_stride.

ROCCC is built on the SUIF2 [3] and Machine-SUIF [4] platforms. We have added new analysis and optimization passes to SUIF2 and Machine-SUIF that target FPGAs. We rely on commercial tools to synthesize the VHDL code generated by our compiler.

Using regular C labels to identify individual loops in a loop nest, the programer specifies which loop-level transformations are to be applied to a loop or loop nest. ROCCC provides the user with predefined and parameterized optimization packages such as unroll, tile, generate systolic array, etc. These packages define the type of loop-level transformation that the user wants to apply to a given loop-nest to be parallelized. The contents and the inner workings of the individual packages are transparent to the user.

\section{SYSTOLIC ARRAY GENERATION FOR WAVEFRONT ALGORITHMS}

Wavefront algorithms are common in bioinformatics and in several other fields such as data-mining and many string algorithms. These algorithms operate over a 2D matrix, where the computation of each cell in the matrix is dependent upon the completion of the computation in its three neighboring cells that are the north, northwest and the west cells. This dependency structure would only allow a single set of diagonal elements of the matrix to be computed at any time. Furthermore, the data bitwidth required by several wavefront algorithms is often much smaller than a standard word-size, leading to narrower customized data paths.

There has been substantial amount of work $[5,6,7,8,9]$ in implementing various forms of wavefront algorithms on FPGAs over the past 10 years or so, however all of these refer to hand-coded HDL. In this section we describe the compiler transformations implemented in ROCCC which map wavefront algorithm codes in $\mathrm{C}$ onto FPGAs.

\subsection{The Smith Waterman Algorithm}

The Smith-Waterman(SW)[1]is a wavefront algorithm example commonly used in bioinformatics. SW is an exact and very computationally expensive sequence alignment algorithm, which computes a matching score of two strings - $S$ and $T$ of sizes $N$ and $M$ respectively - using insertions and deletions. The strings, $S$ and $T$, are formed from a finite alphabet. The cardinality of that alphabet is four for DNA (and RNA) strings and 20 for protein strings.

The algorithm has three steps: the initialization, matrixfill and the trace back. Among the three stages matrix-fill is the one that is most computationally expensive and the hardest to parallelize. It is a wavefront dynamic programming algorithm on a $2 \mathrm{D}$ matrix. Once the first row and the first column of the matrix are initialized, a simple local rule is used to update cell $(i, j)$. Entry $(i, j)$ represents the total score, or edit distance, of aligning a prefix of $S$ of size $i$ against a prefix of $T$ of size $j$. The trace back stage is used to identify the location of the best match if a match is found. However, in the vast majority of runs no match is found. The most commonly used SW computation scheme comes in the following format:

$$
d=\min \begin{cases} \begin{cases}a & \text { if } S_{i}==T_{i} \\ a+\text { substitution } & \text { if } S_{i} !=T_{i}\end{cases} \\ b+\text { insertion } \\ c+\text { deletion }\end{cases}
$$

In the above formula $d$ is the cell being computed, $a$ is the cell's northwest, $b$ is its north and $c$ is the cell's west neighbors. The most typical adjustment scores for the SW implementation on DNAs are insertion and deletion scores being set to 1 and the substitution score being set to 2 . This particular choice of scores would lead into a special property of the SW algorithm, proven in [10], that the horizontal and vertical neighbors would always differ by \pm 1 from one another and are guaranteed to be within \pm 1 of their neighbour cell $a$. This property leads into the fact that transferring only the two low order bits among processing elements within the systolic array would be enough for any length string comparison.

\subsection{Systolic Array Generation in ROCCC}

The outer loop in the $\mathrm{C}$ code shown in Figure 1 is unrolled to form the processing elements of the systolic array cells. The unrolling factor defines the number of processing elements. To optimize for performance, the code should be unrolled along the shorter edge allowing the elements along the longer edge to be streamed in and the hardware systolic array to compute the 2D matrix in bands of length equal to the length of the systolic 
array as illustrated in Figure 5. If we assume that the length of the systolic array is $k$ and the length of the shorter sequence is $m$, this arrangement will require $\operatorname{ceil}(m / k)$ systolic arrays to be processed on the hardware one after another, where the values generated by one systolic array are used to initialize the computation of the next one.

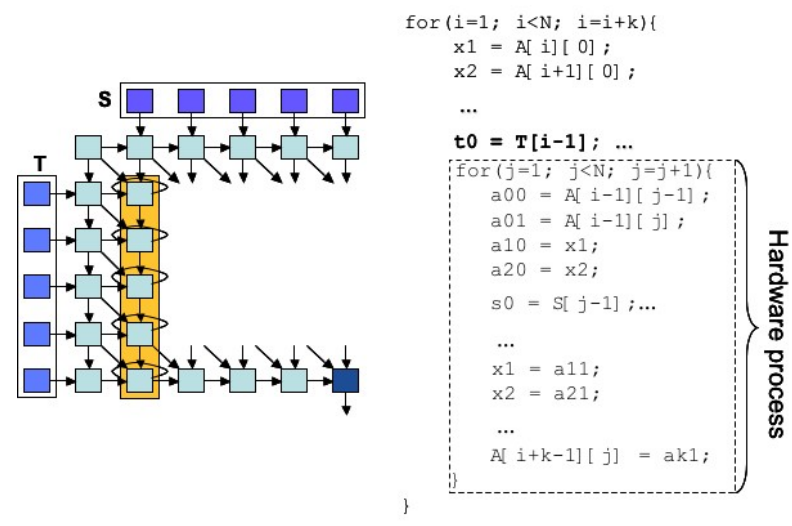

Fig. 3. Code to be mapped to hardware after loop unrolling, scalar replacement, feedback reference elimination and loop invariant code motion

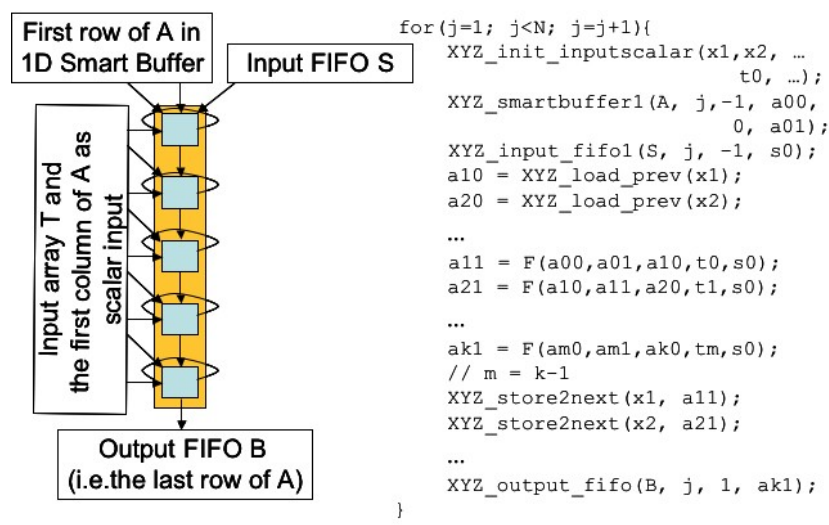

Fig. 4. Generated datapath

Next, the unrolled loop is scalar replaced. Scalar replacement decouples the memory accesses from the actual computation, hence generating a memory access free datapath preceeded by all the array reads and followed by all array stores. The array feedback elimination pass is the major pass that generates the systolic array description at the HLL level. It takes in the scalar replaced code and analyzes the type of dependency therein for every load-store pair. It determines whether an array address stored into during a given iteration is read again the following iteration. Such load-store pairs are replaced with scalar temporaries and any initialization code is copied above the innermost loop. Later, these temporaries are replaced by on-chip registers whose values are computed and kept on-chip to be used by future iterations. The number of on-chip regis-

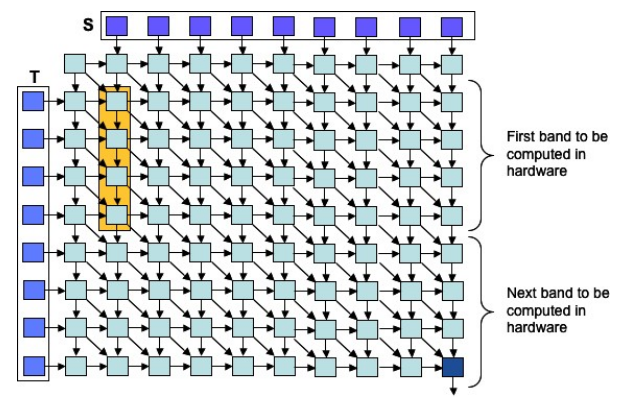

Fig. 5. Systolic array computation in bands

ters needed for a given application is linear in the amount of the unrolling factor.

Figure 3 shows the code after loop unrolling, scalar replacement, feedback reference elimination and loop invariant code motion. Figure 4 shows the final output of the high level optimization passes of ROCCC. The ROCCC_loadprev and ROCCC_store 2 next are macros internal to our compiler. These two macros are inserted into the final code to reflect the effects of the eliminated feedback load/store pairs. ROCCC_load_prev loads a value from the iteration before and $R O C C C$ store $2 n e x t$ saves its input to be read during the next iteration. Both macros are translated into special machine instructions defined in MachineSUIF, followed by VHDL code at later stages. In the generated code, both macros read/write from/to on-chip registers.

In the unaltered wavefront code (Figure 1) each cell computation requires at least three array reads from neighbours and one write. Once unrolled $k$ times, out of a total of $4 k$ memory accesses per loop iteration only a total of three per iteration are left in the resultant code. $4 k-3$ memory accesses are eliminated through the array feedback elimination pass and transferred through wires and on-chip registers. These figures does not include the $1 k$ reads from $T$, which are lifted above the $j$ loop due to invariant code motion, as well as any reads from $S$.

\section{PERFORMANCE EVALUATION}

From the C implementation of SW, ROCCC automatically generates a systolic array implementation of the algorithm as described in the previous section. Our target is the Xilinx Virtex 4 LX200 FPGAs on the SGI RASC RC100 blades [11]. SGI RASC RC100 blade contains two Xilinx Virtex 4 LX200 FPGAs with up to 80MBs of QDR SRAM memory in four modules. Each FPGA is connected to two QDR SRAM memory modules with a bandwidth of $128 \mathrm{bits} / \mathrm{cycle}$ at each connection. These FPGAs operate seamlessly with the SGI Altix Servers [12], which enables the applications to be run part on the highperformance server and part on the RASC blades.

Table 1 compares the performance of the code on an Intel Xeon $2.8 \mathrm{GHz}$ with Hyperthreading and on an Intel Itanium $1.5 \mathrm{GHz}$ to the performance of our ROCCC generated code on the Virtex 4 LX200. The code for the Xeon and Itanium are compiled using the GCC 4.0.2 and the ICC 9.1 compilers respectively. The CPU data is based on the average of five 
International Conference on Field Programmable Logic and Applications. Heidelberg, Germany, September 2008 (FPL'08).

Table 1. Throughput evaluation of Smith-Waterman codes in cell updates per second (CUPS)

\begin{tabular}{|l|r|r|r|}
\hline Platform & Speed & GCUPS & Speedup \\
\hline Intel Xeon & $2.8 \mathrm{GHz}$ & 0.049 & 1 \\
\hline Intel Itanium-2 & $1.5 \mathrm{GHz}$ & 0.084 & 1.7 \\
\hline 200-cells, $2.8 \%$ area & $191 \mathrm{MHz}$ & 38.20 & 780 \\
\hline 512-cells, 6.9\% area & $188 \mathrm{MHz}$ & 96.25 & 1,964 \\
\hline 1024-cells, $17 \%$ area & $174 \mathrm{MHz}$ & 178.65 & 3,646 \\
\hline
\end{tabular}

actual runs and computed as first timing the execution of the candidate loop that is actually mapped onto the hardware and then dividing the measured time by the number of computed cells. FPGA data is based on VHDL simulation (using Xilinx ISE 8.2) with the area and clock frequency obtained after place and route. The performance is measured in giga cell-updates per second (GCUPS) as in the literature for SW algorithms.

So far we have experimented with a systolic array size of up to 1024 cells. ROCCC generated 1024-cell systolic array occupies $17 \%$ of the area of the Virtex 4 LX200 FPGA and achieved a throughput of 178.65 GCUPS. To fill up $70 \%$ of the FPGA area ${ }^{1}$, we can run up to 5 copies of the 1024-cell systolic array (for DNAs) simultaneously on an FPGA, which results in a total speedup of around 10,630 over the Itanium and 18,230 over the Xeon respectively. For the 512 and 200 cell systolic arrays, the throughputs obtained were 96.5 and 38.2 GCUPS while the circuit area was only $6.9 \%$ and $2.8 \%$ of the entire FPGA area respectively.

The ROCCC compiler-generated systolic array code required one element of the input array $S$ and one value from the starting row of the 2D matrix to be fetched onto the FPGA every cycle. Assuming that the sequences under analysis are DNA nucleotides (represented using 2 bits per value), the bandwidth requirement is four bits/cycle. For the five copies of 1024-cell systolic arrays executing simultaneously, the entire computation would require a total of 20 bits/cycle which is well within the capabilities of our target platform.

Our work is the only compiled HDL implementation from $\mathrm{C}$ available. ROCCC generated SW circuit fits 1024 cells to $17 \%$ of the available FPGA area. Our implementation does not require reconfiguration. Circuit is configured once. The circuit reads the query in as a parameter from outside in the start and the database entries are streamed in one element per cycle after circuit's initialization. Analyzing the area growth rates of our circuit from 200 to 512 to 1024 , we expect to fit around 3000 cells on our target FPGA with a throughput estimate of over 500 GCUPS.

\section{CONCLUSION}

In this paper we have presented the high-level transformations performed by ROCCC that allows the automatic generation of

\footnotetext{
${ }^{1}$ The performance of a circuit degrades rapidly when its area exceeds about $80 \%$ of the FPGA area, thus in an efficient FPGA design, typically the maximum used area can not exceed $75 \%-85 \%$
}

systolic arrays from waverfont algorithms in C. To our knowledge, there is currently no other C-to-VHDL compiler that can do the systolic array generation transformation. We used Smith-Waterman, a highly computationally intensive bioinformatics sequence alignment algorithm, to evaluate the generated systolic array. We report a throughput increase by over $3,000 \mathrm{X}$ over a $2.8 \mathrm{GHz}$ Xeon.

\section{REFERENCES}

[1] T. Smith and M. Waterman, "Identification of common molecular subsequences.” J Mol Biol, vol. 147, no. 1, pp. 195-197, 1981.

[2] B. Buyukkurt, Z. Guo, and W. Najjar, "Impact of loop unrolling on area, throughput and clock frequency in roccc: $\mathrm{C}$ to vhdl compiler for impact of loop unrolling on area, throughput and clock frequency in roccc: $\mathrm{C}$ to vhdl compiler for fpgas," in Proc. International Workshop On Applied Reconfigurable Computing, 2006.

[3] G. Aigner, A. Diwan, D. L. Heine, M. S. Lam, D. L. Moore, B. R. Murphy, and C. Sapuntzakis, An Overview of the SUIF2 Compiler Infrastructure, Computer Systems Laboratory, Stanford University.

[4] M. D. Smith and G. Holloway, An introduction to Machine SUIF and its portable libraries for analysis and optimization, Division of Engineering and Applied Sciences, Harvard University.

[5] S. Dydel and P. Bala, "Large scale protein sequence alignment using FPGA reprogrammable logic devices," in Field Programmable Logic and Application. Springer Berlin / Heidelberg, 2004, pp. 23-32.

[6] K. Puttegowda, W. Worek, N. Pappas, A. Dandapani, P. Athanas, and A. Dickerman, "A run-time reconfigurable system for gene-sequence searching," in VLSID '03: Proceedings of the 16th International Conference on VLSI Design, 2003, pp. 561-566.

[7] R. Jacobi, M. Ayala-Rincon, L. Carvalho, C. Llanos, and R. Hartenstein, "Reconfigurable systems for sequence alignment and for general dynamic programming." Genet Mol Res, vol. 4, no. 3, pp. 543-552, 2005.

[8] H.-Y. Liao, M.-L. Yin, and Y. Cheng, "A parallel implementation of the Smith-Waterman algorithm for massive sequences searching," in Proceedings of the 26th Annual International Conference of the IEEE Engineering in Medicine and Biology Society, 2004, pp. 2817-2820.

[9] D. Hoang, "A systolic array for the sequence alignment problem," Technical Report, vol. CS-92-22, 1992.

[10] R. Lipton and D. Lopresti, "A systolic array for rapid string comparison," 1985, pp. 363-376.
[11] SGI RASC RC100 Blade, http://www.sgi.com/products/rasc/datasheets.html.

[12] SGI Altix family, http://www.sgi.com/products/servers/altix/. 